\title{
The effect of obstructive jaundice on the sensitivity of intravenous anesthetic of remimazolam: study protocol for a controlled multicenter trial
}

Wen Liu ${ }^{1 \dagger}$, Bin Yang ${ }^{2 \dagger}$, Jun-Wei Ji ${ }^{1}$, Hua Yang ${ }^{1}$, Hong-Hao Song ${ }^{3 *}$ (D, Hai-Bo Qiu ${ }^{3 *}$ and Jin-Chao Song ${ }^{1 *}$

\begin{abstract}
Background: It is well known that obstructive jaundice could affect the pharmacodynamics of some anesthetics, and the sensitivity of some anesthetics would increase among icteric patients. Remimazolam is a new ultra-shortacting intravenous benzodiazepine sedative/anesthetic, which is a high-selective and affinity ligand for the benzodiazepine site on the GABAA receptor. However, no study has reported the pharmacodynamics of remimazolam in patients with obstructive jaundice. We hypothesize that obstructive jaundice affects the pharmacodynamics of remimazolam, and the sensitivity of remimazolam increases among icteric patients.

Methods/design: The study will be performed as a prospective, controlled, multicenter trial. The study design is a comparison of remimazolam requirements to reach a bispectral index of 50 in patients with obstructive jaundice versus non-jaundiced patients with chronic cholecystitisor intrahepatic bile duct stones. Remimazolam was infused at $6 \mathrm{mg} / \mathrm{kg} / \mathrm{h}$ until this endpoint was reached.

Discussion: Remimazolam could be suitable for anesthesia of patients with obstructive jaundice, because remimazolam is not biotransformed in the liver. Hyperbilirubinemia has been well-described to have toxic effects on the brain, which causes the increasing of sensitivity to some anesthetics, such as desflurane, isoflurane, and etomidate. Furthermore, remimazolam and etomidate have the same mechanism of action when exerting an anesthetic effect. We aim to demonstrate that obstructive jaundice affects the pharmacodynamics of remimazolam, and the dose of remimazolam when administered to patients with obstructive jaundice should be modified.
\end{abstract}

Trial registration: Chinese Clinical Trial Registry ChiCTR2100043585. Registered on 23 February 2021

Keywords: Pharmacodynamics, Remimazolam, Obstructive jaundice

\footnotetext{
*Correspondence: Honghao1995@163.com; roverqiu1978@163.com; sjch2013@163.com

'Wen Liu and Bin Yang contributed equally to this work.

${ }^{3}$ Department of Anesthesiology, Eastern Hepatobiliary Surgery Hospital,

Second Military Medical University, Changhai Rd., No. 225, Shanghai, China

'Department of Anesthesiology, Shidong Hospital of Shanghai, University of

Shanghai for Science and Technology, Shiguang Rd., No. 999, Shanghai,

China

Full list of author information is available at the end of the article
}

C The Author(s). 2022 Open Access This article is licensed under a Creative Commons Attribution 4.0 International License, which permits use, sharing, adaptation, distribution and reproduction in any medium or format, as long as you give appropriate credit to the original author(s) and the source, provide a link to the Creative Commons licence, and indicate if changes were made. The images or other third party material in this article are included in the article's Creative Commons licence, unless indicated otherwise in a credit line to the material. If material is not included in the article's Creative Commons licence and your intended use is not permitted by statutory regulation or exceeds the permitted use, you will need to obtain permission directly from the copyright holder. To view a copy of this licence, visit http://creativecommons.org/licenses/by/4.0/. The Creative Commons Public Domain Dedication waiver (http://creativecommons.org/publicdomain/zero/1.0/) applies to the data made available in this article, unless otherwise stated in a credit line to the data. 


\section{Background}

Obstructive jaundice is defined as the retention of bile components and bile after intrahepatic or extrahepatic bile duct obstruction [1]. Patients with obstructive jaundice are prone to acute renal failure, hypotensive shock, sepsis, and multiple organ dysfunction in the perioperative period $[2,3]$.

Remimazolam is a new, fast-onset, and ultra-shortacting intravenous sedative/anesthetic [4], which is a high-selective and affinity ligand for the benzodiazepine site on the GABAA receptor [5] and rapidly metabolized by tissue esterases to an inactive metabolite $[6,7]$. In recent years, there has been a growing interest in the use of remimazolam in general anesthesia and procedural sedation [4]. It is well known that obstructive jaundice could affect the pharmacodynamics of some anesthetics, and the sensitivity of some anesthetics would increase among icteric patients. We have found that patients with obstructive jaundice are more sensitive to etomidate [8]. In the meanwhile, remimazolam and etomidate have the same mechanism of action when exerting an anesthetic effect, which suggests that patients with obstructive jaundice may also be more sensitive to remimazolam.

\section{Methods/design}

\section{Study settings}

The study is designed as a prospective, controlled, multicenter trial. It is registered in the Chinese Clinical Trial Registry (ChiCTR2100043585. Date of registration: February 23,2021$)$. This study mainly aims to investigate whether obstructive jaundice affects the pharmacodynamics of remimazolam and whether the sensitivity of remimazolam increases among icteric patients. It is reported following the SPIRIT reporting guidelines [9]. The sponsor of this trial is the Department of Anesthesiology, Shidong Hospital of Shanghai, University of Shanghai for Science and Technology. The sponsor is responsible for the design, collection, management, analysis, and interpretation of the data; writing; and the decision to submit the report for publication. The study is supported by the Yangpu District GoodDoctor Program funding and The Science and Technology Commission and Health Committee of Yangpu District, Shanghai (YPM202105). Figure 1 shows the SPIRIT checklist that we follow in this report.

\section{Participants}

\section{Number of patients needed}

We plan to divide the patients into the obstructive jaundice group (total bilirubin (TBL) value $>17.1 \mu \mathrm{mol} / \mathrm{L}$ ) and the control group (TBL $<17.1 \mu \mathrm{mol} / \mathrm{L}$ ) based on their diagnosis and TBL value. The primary endpoint is the difference in the requirement of remimazolam.

The group sample size was calculated based on the differences in remimazolam requirement to reach BIS value $\leq 50$ longer than $5 \mathrm{~s}$ in our previous study, in which the mean remimazolam requirement was $0.13 \pm 0.04 \mathrm{mg} / \mathrm{kg}$ $(n=6)$ in the obstructive jaundice group and $0.16 \pm 0.04$ $\mathrm{mg} / \mathrm{kg}(n=6)$ in the non-obstructive jaundice group, The following formula: $n=15.7 / \mathrm{ES}^{2}+1$, where ES = effect size $=$ (difference between the groups)/(mean of the SD between the groups), with $\alpha=0.05$ and power $=0.8$, was used to determine that the study would be adequately powered with

\begin{tabular}{|c|c|c|c|c|c|c|c|c|c|c|c|c|c|c|}
\hline \multirow[b]{3}{*}{ TIMEPOINT } & \multicolumn{14}{|c|}{ STUDY PERIOD } \\
\hline & \multirow{2}{*}{$\begin{array}{c}\text { Enrolment } \\
-\mathrm{t}_{1}\end{array}$} & \multirow{2}{*}{$\begin{array}{c}\text { Allocation } \\
0\end{array}$} & \multicolumn{11}{|c|}{ Post-allocation } & \multirow{2}{*}{$\begin{array}{c}\text { Close- } \\
\text { out }\end{array}$} \\
\hline & & & $\mathrm{t}_{1}$ & $\mathrm{t}_{2}$ & $\mathrm{t}_{3}$ & $\mathrm{t}_{4}$ & t5 & $t_{6}$ & $\mathrm{t}_{7}$ & $\mathrm{t}_{8}$ & t9 & $\mathrm{t}_{10}$ & $\mathrm{t}_{11}$ & \\
\hline ENROLMENT: & & & & & & & & & & & & & & \\
\hline General information & $x$ & & & & & & & & & & & & & \\
\hline Eligibility screen & $x$ & & & & & & & & & & & & & \\
\hline Informd consent & $x$ & & & & & & & & & & & & & \\
\hline Allocation & & $x$ & & & & & & & & & & & & \\
\hline INTERVENTIONS: & & & & & & & & & & & & & & \\
\hline Remimazolam sedation & & & $\leftarrow$ & & & $\rightarrow$ & & & & & & & & \\
\hline Sufentanil/cisatracurium & & & & & & & $\leftarrow$ & $\rightarrow$ & & & & & & \\
\hline Sevoflurane maintenance & & & & & & & & & $\leftarrow$ & - & & & $\rightarrow$ & \\
\hline ASSESSMENT: & & & & & & & & & & & & & & \\
\hline BIS & & & $x$ & $x$ & $x$ & $x$ & $x$ & $x$ & $x$ & $x$ & $x$ & $x$ & $x$ & $x$ \\
\hline Time interval & & & & & & $x$ & & & & & & & & $x$ \\
\hline Hemodynamic variables & & $x$ & $x$ & $x$ & $x$ & $x$ & $x$ & $x$ & $x$ & $x$ & $x$ & $x$ & $x$ & $x$ \\
\hline Outcome variables & & & & & & $x$ & & & & & & & & $x$ \\
\hline
\end{tabular}

Fig. 1 Schedule of enrollment, intervention, and assessment according to the Standard Protocol Items: Recommendations for Interventional Trials (SPIRIT) statement 
$n=29$ per group. Considering a dropout rate of $10 \%$, the estimated sample size will be at least 32 patients per group; thus, a total of 64 patients will be needed.

\section{Eligibility}

The study takes place at three centers: the Department of Anesthesiology, Shidong Hospital of Shanghai, University of Shanghai for Science and Technology; Department of Anesthesiology, Eastern Hepatobiliary Surgery Hospital, Second Military Medical University; and Department of Anesthesiology, Chongqing University Cancer Hospital beginning March 2021 to March 2022. Eligible patients for participation in this clinical trial are 32 patients with obstructive jaundice (serum TBL > $17.1 \mu \mathrm{mol} / \mathrm{L}$ ) secondary to neoplasm of the bile duct or the head of the pancreas and 32 non-jaundiced patient controls with chronic cholecystitisor intrahepatic bile duct stones. All patients enrolled in this clinical trial are aged 18 to 75 years, American Society of Anesthesiologists (ASA) grades II to III, and given written informed consent, who are scheduled to undertake surgery.

\section{Exclusion criteria}

Patients are excluded if the following criteria appear in their medical history:

- Age range $<18$ years or $>75$ years

- ASA physical status I, IV, or V

- Weight beyond $\pm 20 \%$ of ideal

- Allergic reaction to the planned medication

- History of psychological problems or psychiatric disease

- Using any form of analgesic or neuromodulating medications

- Known or suspected cardiac, pulmonary, renal, or metabolic disease

The schedule of enrollment, intervention, and assessment is reported according to the SPIRIT statement (Fig. 1).

The number of excluded patients and the reasons for their exclusion will be reported according to the SPIRIT statement.

\section{Consent}

Written consent was obtained from all patients. The patient's history and current health status are screened during the standard anesthesia evaluation before the surgery. The investigators use anesthesia pre-assessment sheets to screen patients for inclusion and exclusion criteria. Inclusion will not be finalized until the patient signs the informed consent on the day of surgery.

If patients refuse to participate in the study, they will be sedated with propofol according to the anesthetic standards. The investigator or physician who examines the subject may decide to remove the subject from the study if the subject has an emergency medical problem (allergic reaction or acute health problem).

\section{Ethical approval}

The trial is approved by the Committee on Ethics of Biomedicine Research, Shidong Hospital Affiliated to University of Shanghai for Science and Technology (YPSD KY2020-004-010).

\section{Allocation and allocation concealment}

Patients are allocated to the obstructive jaundice group or the control group based on the TBL values after they sign the informed consent form. Because obstructive jaundice is easy to distinguish from the appearance of skin color, it is difficult to double-blind. However, the anesthesiologist assistant is blind to the grouping or induction method but only records the data through a local area network (LAN) in the next room.

\section{Intervention}

After $8 \mathrm{~h}$ of fasting, the patients without premedication will be brought into a quiet operating room where a cannula was inserted into the right internal jugular vein under local anesthesia for infusion of remimazolam and liquid. Radial artery catheterization was placed to measure invasive arterial blood pressure. Heart rate (HR), invasive blood pressure (IBP), electrocardiogram (ECG), end-tidal carbon dioxide $\left(\mathrm{ETCO}_{2}\right)$, and oxyhemoglobin saturation $\left(\mathrm{SpO}_{2}\right)$ are routinely monitored during the whole process of research (Philips HP Viridia24/26 M1205A). Apply the BIS sensor (BIS ${ }^{\text {rix } X P}$ sensor) as recommended by the manufacturer. The patients are asked to keep their eyes closed and covered with gauze to avoid any interference with sound and light stimulation. The temperature of the room is controlled at $23{ }^{\circ} \mathrm{C}$.

The study is designed to record the remimazolam requirement with BIS of 50 as the endpoint. The patients will be treated with remimazolam at a rate of $6 \mathrm{mg} / \mathrm{kg} / \mathrm{h}$ by a Graseby 3500 syringe pump (SIMS Graseby Ltd., Herts, UK) until the BIS is $\leq 50$ longer than $5 \mathrm{~s}$. The assistant anesthesiologist, who is not aware of the study group, observes the vital signs and BIS values in the next room via the LAN, determines the endpoint of titration, and records the dosage of remimazolam used and the time interval between the start and end of the infusion. Then, the patients will be given $0.4-0.6 \mu \mathrm{g} / \mathrm{kg}$ sufentanil and $0.2 \mathrm{mg} / \mathrm{kg}$ cisatracurium, and endotracheal intubation will be performed 3 min later for anesthesia induction. Anesthesia is maintained with sevoflurane (1.5$2.5 \%)$ at an appropriate standard for surgical procedures. Hemodynamic data are collected and recorded at relevant points during the peri-intubation period. 


\section{Primary objective}

\section{Definition of primary endpoint}

The primary endpoint of the study-reflecting the change of sensitivity of remimazolam in patients with obstructive jaundice-is remimazolam requirement with BIS of 50 as the endpoint.

\section{Assessment of primary endpoint}

We will record the requirement of remimazolam and the time interval between the start and end of the infusion.

\section{Secondary objectives}

\section{Definition of secondary endpoints}

Secondary endpoints focus on hemodynamic stability and safety, which is reflected in the number of cardiovascular events and the average percent change to baseline in the mean arterial pressure and heart rate.

\section{Assessment of secondary endpoint}

Hemodynamic data at the designated time points will be recorded during the perintubation period.

\section{Safety management and adverse event}

Researchers make sure that emergency equipment is working throughout the process. An adverse event refers to any untoward medical occurrence which happens during the trial. Adverse events include but are not limited to respiratory depression or cardiocirculatory instability. All adverse events will be treated immediately. If spontaneous ventilation is insufficient $\left(\mathrm{SpO}_{2}<92 \%\right)$, auxiliary mask ventilation is given to the patients when necessary to maintain $\mathrm{ETCO}_{2}$ between 34 and 45 mmHg. Cardiovascular events should be handled promptly, with 5-15 mg ephedrine given if the patient's blood pressure is below $60 \mathrm{mmHg}$ and $5 \mathrm{mg}$ atropine given if the patient's heart rate is below $50 \mathrm{bpm}$. The type of adverse event, likely cause, and treatment will be documented and discussed in data monitoring committee (DMC) meetings.

\section{Data collection and data management}

Before surgery, general information and the surgical methods of the patients are investigated, and indexes such as TBL, bile acid, albumin, alanine aminotransferase (ALT), aspartate aminotransferase (AST), and alkaline phosphatase (ALP) are recorded. The requirement of remimazolam until the patient's BIS value reaches 50 and the time interval from the start to the end of infusion are recorded. Pulmonary and cardiovascular vital signs are recorded electronically throughout the procedure, including $\mathrm{SpO}_{2}$ measured by a pulse oximeter, $\mathrm{HR}$, IBP, RR, and $\mathrm{ETCO}_{2}$. Data will be collected on paper case report forms (CRFs) by an anesthesiologist assistant blinded to the group allocation in each center.

\section{Data monitoring}

The data monitoring committee, composed of statisticians, representatives from the ethics committee, and principal investigators from each center, will be responsible for data monitoring. The members of the DMC are independent of the sponsors. Written reports on trial progress will be submitted to the committee quarterly. Cases of unexpected scenarios and adverse events will be discussed at committee meetings.

\section{Statistical analysis}

Statistical analyses will be performed by an independent statistician using the SPSS 19.0 statistical software. The measurement data of normal distribution will be presented as mean \pm standard deviation. Independent sample $T$ test or rank-sum test will be used for comparison between the groups, and multiple linear regression analysis will be used to test the relationship between the dosage of remimazolam and TBL, TBA, ALB, AST, and ALT.

\section{Discussion}

Obstructive jaundice may result in hepatic cell damage and hepatosis through various mechanisms [10]. Alanine aminotransferase, aspartate aminotransferase, and alkaline phosphatase increased in obstructive jaundice patients $[8,11]$. The drug-metabolizing enzyme systems may be impaired in patients with obstructive jaundice. Plasma clearance of drugs such as dexmedetomidine and rocuronium, which are metabolized and excreted by the liver, is decreased significantly in patients with obstructive jaundice. Furthermore, cardiovascular abnormalities have been well known to occur in obstructive jaundice patients for many years [12-14]. It was reported that internal biliary drainage produces an improvement in hemodynamics [15].

Remimazolam could be suitable for anesthesia of patients with obstructive jaundice, because remimazolam is not biotransformed in the liver. Schüttler et al. reported that remimazolam has a high clearance $(1.15 \pm 0.12 \mathrm{~L} /$ min), a small steady-state volume of distribution (35.4 \pm $4.2 \mathrm{~L})$, and a short terminal half-life $(70 \pm 10 \mathrm{~min})$. The simulated context-sensitive halftime after an infusion of $4 \mathrm{~h}$ was $6.8 \pm 2.4 \mathrm{~min}$ [16]. It was reported that remimazolam, which did not accumulate after prolonged infusion, could be used for the maintenance of general anesthesia in many nations $[4,17]$. Furthermore, remimazolam was characterized by moderate hemodynamic side effects [16]. A multicenter, randomized, doubleblind, parallel-group trial demonstrated remimazolam's safety and efficacy in vulnerable patients (ASA class III) 
undergoing elective general surgery [18]. All these profiles of remimazolam determine its position in anesthesia for patients with obstructive jaundice, in which patients the duration of the operation is often relatively long and the hemodynamics is relatively unstable.

Hyperbilirubinemia has been well-described to have toxic effects on the brain. Histologic evidence of neurologic damage in the brain, such as atrophy and pyknosis of nerve cells, ghost cells, and neuronophagia, was observed in a well-designed canine model of cholestasis secondary to bile duct resection. Furthermore, neurologic damage is more widespread as the duration of hyperbilirubinemia is prolonged [19]. It has been reported that interaction of unconjugated bilirubin with synaptosomal membrane vesicles results in oxidative injury, calcium intrusion, and loss of membrane asymmetry and functionality, thus potentially contributing to the pathogenesis of encephalopathy by hyperbilirubinemia [20]. Acute hyperbilirubinemia results in presynaptic neurodegeneration at a central glutamatergic synapse [21]. Unconjugated bilirubin could also impair the release and uptake of the neurotransmitter glutamate, indicating possible excitotoxic damage [22, 23]. It has been reported that altered neurotransmission could occur in the brains of cholestatic patients. Some evidence suggests that fatigue and pruritus accompanying with cholestasis may be the consequences of a central mechanism, increased opioidergic neurotransmission [24, 25], and defective serotoninergic neurotransmission [26-28]. Consequently, all these changes in patients with obstructive jaundice may, at least in part, induce the increased sensitivity to anesthetics and the reduction of anesthetic requirements.

Our previous study demonstrates that the MACawake of desflurane was significantly decreased in obstructive jaundice patients compared with non-jaundiced controls; furthermore, there is a highly inverse relation between the MACawake of desflurane and the concentration of serum total bilirubin [29]. We also demonstrated that patients with obstructive jaundice had an increased sensitivity to isoflurane, more bradycardia and hypotension during anesthesia induction and maintenance, and a prolonged recovery time compared with non-jaundiced patients [30].

In our other research, it was shown that the etomidate requirement was significantly decreased in patients with obstructive jaundice, and there was a significant negative correlation between serum total bilirubin and etomidate requirement [8]. Etomidate is a pure hypnotic GABA agonist [31-33]. Shi et al. demonstrated that bilirubin potentiates inhibitory synaptic transmission in the lateral superior olive neurons of rats, and the potentiation of depolarizing GABA/glycinergic transmission by bilirubin could underlie bilirubin excitotoxicity $[34,35]$.
It just so happens that the remimazolam we are discussing is also a high-affinity and selective ligand for the benzodiazepine site on the GABAA receptor. Moreover, remimazolam does not show selectivity between GABAA receptor subtypes [5]. In other words, remimazolam and etomidate have the same mechanism of action when exerting an anesthetic effect. Patients with obstructive jaundice are more sensitive to etomidate [8], which suggests that patients with obstructive jaundice may also be more sensitive to remimazolam.

In conclusion, the toxic effects of hyperbilirubinemia on the central nervous system, such as histologic evidence of neurologic damage, altered neurotransmission in the brain, presynaptic neurodegeneration at the central glutamatergic synapses, and impairment of the release and uptake of the neurotransmitter glutamate, may induce the increased sensitivity to anesthetics and the reduction of anesthetic requirements.

The aim of our trial is to show that obstructive jaundice affects the pharmacodynamics of remimazolam, and the sensitivity of remimazolam increases among icteric patients; thereby, anesthesiologists caring for patients with obstructive jaundice should be alert to the interaction of bilirubin and remimazolam sensitivity.

\section{Trial status}

The first patient was included on 1 March 2021. We expect to finalize the study in March 2022.

\section{Conflict of interest statement}

There are no commercial or financial interests involved in this work.

\section{Acknowledgements \\ The authors are grateful for the financial supports from the Yangpu District Good-Doctor Program.}

Authors' contributions

Wen Liu and Bin Yang are responsible for drafting the manuscript. Jin-Chao Song, Hai-Bo Qiu, and Hong-Hao Song are responsible for the study design. Hua Yang and Jun-Wei Ji are responsible for revising the manuscript. All authors have read and approved the final manuscript.

\section{Funding}

The trial is supported by the Yangpu District Good-Doctor Program and The Science and Technology Commission and Health Committee of Yangpu District, Shanghai (YPM202105).

\section{Availability of data and materials \\ Not applicable.}

\section{Declarations}

\section{Ethics approval and consent to participate}

The trial is approved by the Committee on Ethics of Biomedicine Research, Shidong Hospital Affiliated to University of Shanghai for Science and Technology (YPSDKY2020-004-010). Written consent was obtained from all patients.

Competing interests

The authors declare that they have no competing interests. 


\section{Author details}

${ }^{1}$ Department of Anesthesiology, Shidong Hospital of Shanghai, University of Shanghai for Science and Technology, Shiguang Rd., No. 999, Shanghai, China. ${ }^{2}$ Department of Anesthesiology, Chongqing University Cancer Hospital, Chongqing, China. ${ }^{3}$ Department of Anesthesiology, Eastern Hepatobiliary Surgery Hospital, Second Military Medical University, Changhai Rd., No. 225, Shanghai, China.

\section{Received: 5 May 2021 Accepted: 27 December 2021}

Published online: 08 January 2022

\section{References}

1. Brandoni A, Villar SR, Picena JC, Anzai N, Endou H, Torres AM. Expression of rat renal cortical OAT1 and OAT3 in response to acute biliary obstruction. Hepatology. 2006;43(5):1092-100. https://doi.org/10.1002/hep.21142.

2. Green J, Better OS. Systemic hypotension and renal failure in obstructive jaundice-mechanistic and therapeutic aspects. J Am Soc Nephrol. 1995; 5(11):1853-71. https://doi.org/10.1681/ASN.V5111853.

3. Kimmings AN, van Deventer SJ, Obertop H, Rauws EA, Gouma DJ. Inflammatory and immunologic effects of obstructive jaundice: pathogenesis and treatment. J Am Coll Surg. 1995;181(6):567-81.

4. Keam SJ. Remimazolam: first approval. Drugs. 2020;80(6):625-33. https://doi. org/10.1007/s40265-020-01299-8.

5. Kilpatrick GJ, Mclntyre MS, Cox RF, Stafford JA, Pacofsky GJ, Lovell GG, et al. CNS 7056: a novel ultra-short-acting benzodiazepine. Anesthesiology. 2007; 107(1):60-6. https://doi.org/10.1097/01.anes.0000267503.85085.c0.

6. Wiltshire HR, Kilpatrick GJ, Tilbrook GS, Borkett KM. A placebo- and midazolam-controlled phase I single ascending-dose study evaluating the safety, pharmacokinetics, and pharmacodynamics of remimazolam (CNS 7056): Part II. Population pharmacokinetic and pharmacodynamic modeling and simulation. Anesth Analg. 2012;115(2):284-96. https://doi.org/10.1213/A NE.0b013e318241f68a.

7. Antonik L, Goldwater DR, Kilpatrick GJ, Tilbrook GS, Borkett KM. A placeboand midazolam-controlled phase I single ascending-dose study evaluating the safety, pharmacokinetics, and pharmacodynamics of remimazolam (CNS 7056): Part I. Safety, efficacy, and basic pharmacokinetics. Anesth Analg. 2012;115(2):274-83. https://doi.org/10.1213/ANE.0b013e31823f0c28.

8. Song JC, Sun YM, Zhang MZ, Yang LQ, Tao TZ, Yu WF. The etomidate requirement is decreased in patients with obstructive jaundice. Anesth Analg. 2011;113(5):1028-32. https://doi.org/10.1213/ANE.0b013e31822dac4a.

9. Chan A-W, Tetzlaff JM, Gøtzsche PC, Altman DG, Mann H, Berlin J, et al. SPIRIT 2013 explanation and elaboration: guidance for protocols of clinical trials. BMJ. 2013;346(jan08 15):e7586. https://doi.org/10.1136/bmj.e7586.

10. Mochida S, Ogata I, Hirata K, Ohta Y, Yamada S, Fujiwara K. Provocation of massive hepatic necrosis by endotoxin after partial hepatectomy in rats. Gastroenterology. 1990;99(3):771-7. https://doi.org/10.1016/00165085(90)90967-6.

11. Song JC, Gao H, Qiu HB, Chen QB, Cai MH, Zhang MZ, et al. The pharmacokinetics of dexmedetomidine in patients with obstructive jaundice: a clinical trial. PLoS One. 2018;13(11):e0207427. https://doi.org/1 0.1371/journal.pone.0207427.

12. Ma Z, Zhang Y, Huet PM, Lee SS. Differential effects of jaundice and cirrhosis on beta-adrenoceptor signaling in three rat models of cirrhotic cardiomyopathy. J Hepatol. 1999;30(3):485-91. https://doi.org/10.1016/S01 68-8278(99)80109-3.

13. Lumlertgul D, Boonyaprapa S, Bunnachak D, Thanachaikun N, Praisontarangkul OA, Phornphutkul K, et al. The jaundiced heart: evidence of blunted response to positive inotropic stimulation. Ren Fail. 1991;13(1):1522. https://doi.org/10.3109/08860229109022141.

14. Ljubuncic P, Said O, Ehrlich Y, Meddings JB, Shaffer EA, Bomzon A. On the in vitro vasoactivity of bile acids. Br J Pharmacol. 2000;131(3):387-98. https:// doi.org/10.1038/sj.bjp.0703554.

15. Padillo J, Puente J, Gómez M, Dios F, Naranjo A, Vallejo JA, et al. Improved cardiac function in patients with obstructive jaundice after internal biliary drainage: hemodynamic and hormonal assessment. Ann Surg. 2001;234(5): 652-6. https://doi.org/10.1097/00000658-200111000-00010.

16. Schüttler J, Eisenried A, Lerch M, Fechner J, Jeleazcov C, Ihmsen $H$. Pharmacokinetics and pharmacodynamics of remimazolam (CNS 7056) after continuous infusion in healthy male volunteers: Part I. Pharmacokinetics and clinical pharmacodynamics. Anesthesiology. 2020;132(4):636-51. https://doi. org/10.1097/ALN.0000000000003103.
17. Sheng XY, Liang Y, Yang XY, Li LE, Ye X, Zhao X, et al. Safety, pharmacokinetic and pharmacodynamic properties of single ascending dose and continuous infusion of remimazolam besylate in healthy Chinese volunteers. Eur J Clin Pharmacol. 2020;76(3):383-91. https://doi.org/10.1007/s00228-019-02800-3.

18. Doi M, Hirata N, Suzuki T, Morisaki H, Morimatsu H, Sakamoto A. Safety and efficacy of remimazolam in induction and maintenance of general anesthesia in high-risk surgical patients (ASA Class III): results of a multicenter, randomized, double-blind, parallel-group comparative trial. J Anesth. 2020;34(4):491-501. https://doi.org/10.1007/s00540-020-02776-w.

19. Furukawa Y. Histological changes in the brain due to experimental obstructive jaundice. Nihon Geka Gakkai Zasshi. 1991;92(1):37-45.

20. Brito MA, Brites D, Butterfield DA. A link between hyperbilirubinemia, oxidative stress and injury to neocortical synaptosomes. Brain Res. 2004; 1026(1):33-43. https://doi.org/10.1016/j.brainres.2004.07.063.

21. Haustein MD, Read DJ, Steinert JR, Pilati N, Dinsdale D, Forsythe ID. Acute hyperbilirubinaemia induces presynaptic neurodegeneration at a central glutamatergic synapse. J Physiol. 2010;588(Pt 23):4683-93. https://doi.org/1 0.1113/jphysiol.2010.199778.

22. Silva RF, Rodrigues $C M$, Brites D. Rat cultured neuronal and glial cells respond differently to toxicity of unconjugated bilirubin. Pediatr Res. 2002; 51(4):535-41. https://doi.org/10.1203/00006450-200204000-00022.

23. Silva R, Mata LR, Gulbenkian S, Brito MA, Tiribelli C, Brites D. Inhibition of glutamate uptake by unconjugated bilirubin in cultured cortical rat astrocytes: role of concentration and pH. Biochem Biophys Res Commun. 1999;265(1):67-72. https://doi.org/10.1006/bbrc.1999.1646

24. Bergasa NV, Alling DW, Talbot TL, Swain MG, Yurdaydin C, Turner ML, et al. Effects of naloxone infusions in patients with the pruritus of cholestasis. A double-blind, randomized, controlled trial. Ann Intern Med. 1995;123(3):1617. https://doi.org/10.7326/0003-4819-123-3-199508010-00001.

25. Bergasa NV, Jones EA. The pruritus of cholestasis: potential pathogenic and therapeutic implications of opioids. Gastroenterology. 1995;108(5):1582-8. https://doi.org/10.1016/0016-5085(95)90709-2.

26. Jones EA, Yurdaydin C. Is fatigue associated with cholestasis mediated by altered central neurotransmission? Hepatology. 1997;25(2):492-4. https://doi. org/10.1002/hep.510250239.

27. Swain MG, Maric M. Improvement in cholestasis-associated fatigue with a serotonin receptor agonist using a novel rat model of fatigue assessment. Hepatology. 1997;25(2):291-4. https://doi.org/10.1002/hep.510250206.

28. Burak KW, Le T, Swain MG. Increased midbrain 5-HT1A receptor number and responsiveness in cholestatic rats. Brain Res. 2001;892(2):376-9. https:// doi.org/10.1016/S0006-8993(00)03058-4.

29. Song JG, Cao YF, Yang LQ, Yu WF, Li Q, Song JC, et al. Awakening concentration of desflurane is decreased in patients with obstructive jaundice. Anesthesiology. 2005;102(3):562-5. https://doi.org/10.1097/ 00000542-200503000-00014.

30. Yang LQ, Song JC, Irwin MG, Song JG, Sun YM, Yu WF. A clinical prospective comparison of anesthetics sensitivity and hemodynamic effect among patients with or without obstructive jaundice. Acta Anaesthesiol Scand. 2010;54(7):871-7. https://doi.org/10.1111/j.1399-6576.2010.02222.x.

31. Belelli D, Lambert JJ, Peters JA, Wafford K, Whiting PJ. The interaction of the general anesthetic etomidate with the gamma-aminobutyric acid type A receptor is influenced by a single amino acid. Proc Natl Acad Sci U S A. 1997;94(20):11031-6. https://doi.org/10.1073/pnas.94.20.11031.

32. Desai R, Ruesch D, Forman SA. Gamma-amino butyric acid type A receptor mutations at beta2N265 alter etomidate efficacy while preserving basal and agonist-dependent activity. Anesthesiology. 2009;111(4):774-84. https://doi. org/10.1097/ALN.0b013e3181b55fae.

33. Martin $L$, Oh GH, Orser BA. Etomidate targets alpha5 gamma-aminobutyric acid subtype $A$ receptors to regulate synaptic plasticity and memory blockade. Anesthesiology. 2009;111(5):1025-35. https://doi.org/10.1097/ALN. Ob013e3181 bbc961.

34. Shi HB, Kakazu Y, Shibata S, Matsumoto N, Nakagawa T, Komune S. Bilirubin potentiates inhibitory synaptic transmission in lateral superior olive neurons of the rat. Neurosci Res. 2006;55(2):161-70. https://doi.org/10.1016/j.neures.2006.02.015.

35. Li CY, Shi HB, Wang J, Ye HB, Song NY, Yin SK. Bilirubin facilitates depolarizing GABA glycinergic synaptic transmission in the ventral cochlear nucleus of rats. Eur J Pharmacol. 2011;660(2-3):310-7. https//doi.org/10.1016/j.jphar.2011.03.017.

\section{Publisher's Note}

Springer Nature remains neutral with regard to jurisdictional claims in published maps and institutional affiliations. 